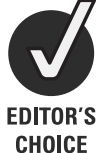

CHOICE
Oncology Department, Old Road Research Campus Building, Oxford, United Kingdom, OX3 $7 D 0$

\section{Correspondence to} Dr Ricky A Sharma, Cancer Research UK-Medical Research Council Gray Institute for Radiation Oncology and Biology, Oncology Department, Old Road Research Campus Building, OX3 7D0, UK; ricky.sharma@ oncology.ox.ac.uk

Accepted 17 May 2012 Published Online First 26 June 2012

\title{
Pathological grading of regression following neoadjuvant chemoradiation therapy: the clinical need is now
}

\author{
Tom P MacGregor, Tim S Maughan, Ricky A Sharma
}

\section{ABSTRACT}

Neoadjuvant chemoradiotherapy for locally advanced rectal cancer has been shown to decrease rates of local recurrence and more than double the rate of sphincterpreserving surgery. There is now compelling evidence that pathological complete response is an independent predictor of likelihood of local recurrence, distal metastases, disease-free and overall survival in locally advanced rectal cancer following neoadjuvant chemoradiotherapy. Pathological regression grading can therefore guide clinical decisions about salvage surgical strategies, adjuvant therapy and long-term surveillance. No universally recognised regression grading system currently exists for pathologists presented with resected tumour specimens following neoadjuvant chemoradiotherapy. The purpose of this review is to highlight the relevance of accurate tumour regression grading in achieving optimal clinical care for patients with rectal cancer.

\section{INTRODUCTION}

The doubling in survival rates in rectal cancer that has been reported over the last 30 years has been driven by several factors. The focus on the anatomy of the rectum and the local spread of the tumour was central to three key improvements: first, the accurate delineation of the involvement of the circumferential margin on pathological examination of the excised specimen; ${ }^{1}$ second, the introduction of total mesorectal excision; ${ }^{2}$ and, third, the preoperative demonstration of the relationship of the tumour to the circumferential resection margin. ${ }^{3} 4$ Earlier diagnosis from the introduction of screening programmes is now shifting the distribution of stage towards increased diagnosis of premalignant neoplasia and early rectal cancer which together now make up $50 \%$ of surgical cases. In this context, neoadjuvant chemoradiotherapy has been increasingly accepted as having a key role in the management of locally advanced rectal cancer (LARC).

This review article focuses on the clinical and histopathological consequences of neoadjuvant chemoradiotherapy in order to make a clinical case for the current need for a consensus on this issue. The aim of the authors is to open the door towards a consistent interpretation of this key early surrogate marker of neoadjuvant radiotherapy in rectal cancer.

\section{THE SHIFT FROM ADJUVANT TO NEOADJUVANT CHEMORADIATION THERAPY \\ Radiotherapy}

Although radiotherapy has been used to treat rectal cancer for more than half a century, it was not until
1988 that Gérard et al demonstrated that longcourse neoadjuvant radiotherapy (34.5 Gy divided into 15 daily doses of 2.3 Gy each) improved both 5 -year survival and local recurrence rates following radical surgery for rectal cancer (T2, T3, T4, NX, M0). ${ }^{5}$ It was subsequently shown that short-course preoperative radiotherapy (SCRT) (25.5 Gy in five fractions) followed by surgery within 1 week decreased local recurrence rates relative to postoperative radiotherapy over 5 weeks. ${ }^{6}$ Allowing for radiobiological assumptions, the cumulative radiation effect of SCRT in 1 week on the tumour is thought to be approximately equivalent to $46 \mathrm{~Gy}$ of long-course radiotherapy if fractions of 2 Gy were given 5 days per week. ${ }^{7}$ The 12-year follow-up results of the Dutch TME trial have shown that, although SCRT given immediately preoperatively decreases the incidence of local recurrence, it does not improve overall survival. ${ }^{8}$

The Stockholm III clinical trial is currently recruiting patients with the aim of directly comparing SCRT and long-course radiotherapy preoperatively and also to determine the optimum timing of surgery following preoperative radiotherapy. ${ }^{9}$ It is a three-armed trial comparing SCRT $(5 \times 5$ Gy) with surgery within 1 week, SCRT with surgery after 4-8 weeks and long-course radiotherapy $(25 \times 2$ Gy) with surgery after $4-8$ weeks. The interim analysis of this trial has shown similar pathological response rates between SCRT with surgery after 4-8 weeks and long-course radiotherapy $(25 \times 2 \mathrm{~Gy})$ with surgery after $4-8$ weeks In the other treatment arm of the clinical trial a trend towards more postoperative complications has been suggested when SCRT is followed immediately (ie, $<4$ weeks) by surgery, but only if that surgery is delayed beyond 10 days after the start of radiotherapy. ${ }^{9}$ The definitive results of this clinical trial, expected in 2014-2015, will demonstrate the clinical differences between two 'radiobiologically equivalent' fractionation regimes and the preferred timing of surgery following radiotherapy.

\section{Neoadjuvant chemoradiation}

The combination of chemotherapy and radiotherapy (ie, chemoradiation) was first found to be more effective than radiotherapy alone in the adjuvant (postoperative) setting for advanced rectal cancer by the Gastrointestinal Tumor Study Group in the USA in 1985, with decreased rates of recurrence and increased time to recurrence when adjuvant chemoradiotherapy with 5 -fluorouracil (5-FU) was compared with adjuvant radiotherapy or surgery alone. ${ }^{10}$ The North Central CCG confirmed 
this effect showing that the addition of 5 -FU to adjuvant radiotherapy improved overall survival by $29 \%$ at 7 years. ${ }^{11}$ More recently, the German Rectal Cancer Study Group has demonstrated that neoadjuvant (preoperative) chemoradiotherapy for LARC decreased rates of local recurrence and resulted in fewer short- and long-term toxic effects than those from postoperative chemoradiotherapy. The treatment regimen used was 50.4 Gy delivered in 1.8 Gy fractions per day, 5 days per week, with a 120-h continuous infusion of 5-FU during the first and fifth weeks of radiotherapy. They also observed that preoperative chemoradiotherapy more than doubled the rate of sphincter-preserving surgery. ${ }^{12}$ The German study was a critical result that reflects a change in international practice away from postoperative chemoradiation to preoperative treatment.

Currently, neoadjuvant chemoradiotherapy is generally considered for all LARC (defined as advanced cT3 or T4; see box 1) with potential circumferential resection margin involvement on pretreatment MRI. In preference to CT scanning, MRI is now considered the gold standard for the preoperative staging of rectal cancer, as evidenced by the impressive results of the MERCURY Study Group (see below). ${ }^{14}$

Neoadjuvant chemoradiotherapy regimens utilise systemic fluoropyrimidines as radiosensitisers, administered concomitantly with high dose radiotherapy. Classically, 5-FU has been used as chemotherapy. ${ }^{10}$ On account of its short half-life in vivo, 5 -FU is optimally delivered as a continuous infusion either over 48-96 h during weeks 1 and 5 of radiotherapy, ${ }^{11}$ or as a low dose continuous infusion for the duration of the course of radiotherapy. ${ }^{15}$ In some countries, the oral fluoropyrimidine, capecitabine, a prodrug of 5-FU, has replaced infusional 5-FU on account of its convenience and, since it can be consumed by the patient on a daily basis from the first to the last day of radiotherapy, potentially providing more consistent radiosensitisation throughout the entire 5-5.5 weeks of radiotherapy. ${ }^{16}{ }^{17}$ Based on known synergy in treating metastatic disease, there is currently interest in the use of combination chemotherapy in neoadjuvant chemoradiation for LARC. Agents under investigation include oxaliplatin and irinotecan ${ }^{18}{ }^{19}$ both known to be radiosensitisers per se, plus monoclonal antibodies such as cetuximab. $^{20}$

Box 1 Summary of the tumour node metastasis (TNM) classification of colorectal cancer ${ }^{13}$

T1: Submucosa

- T2: Muscularis propria

- T3: Subserosa, perirectal tissues

- T4a: Visceral peritoneum

- T4b: Other organs or structures

- N1a: One regional lymph node involved

- N1b: Two to three regional lymph nodes involved

- N1c: Satellites without regional lymph nodes

- N2a: Four to six regional lymph nodes involved

- N2b: Seven or more regional lymph nodes involved

- M1a: Metastasis to one organ

- M1b: Metastases to more than one organ or peritoneum

- y: Prefix indicates staging taking place during/following multimodal therapy

c: Prefix indicates clinical staging

p: Prefix indicates pathological staging
TRG FOLLOWING NEOADJUVANT THERAPY Classification of tumour regression

Tumour regression grading (TRG) as a measurement of response to neoadjuvant radiotherapy was first proposed by Mandard et al in 1994 for use in the assessment of pathological specimens of squamous cell carcinoma of the oesophagus following neoadjuvant chemoradiotherapy. ${ }^{21}$ Mandard and colleagues classified TRG into five grades from TRG 1 (complete regression) to TRG 5 (no regression) based on the presence of residual cancer cells and the degree of fibrotic change. Pathological response was defined as the presence of TRG $1-3$ in the resected specimen, and was shown to be an independent predictor of disease-free survival. $^{21}$

Three years later, Dworak et al reported a system for the grading of regression (GR) following neoadjuvant chemoradiotherapy of rectal tumours. They graded regression from GR 0 (no regression) to GR 4 (complete regression), assessing the resected specimen for tumour mass, fibrotic changes, irradiation vasculopathy and peri-tumourous inflammatory reaction. ${ }^{22}$ This report was followed by the Rectal Cancer Regression Grade (RCRG), which simplified the classification to three levels: RCRG 1: the tumour is either sterilised or only microscopic foci of adenocarcinoma remain; RCRG 2: marked fibrosis, but with macroscopic tumour still present; and RCRG 3: little or no fibrosis in the presence of abundant macroscopic tumour. RCRG 1 and 2 were considered to represent significant tumour regression. $^{23}$

The most recently published classification, the Royal College of Pathologists dataset guidelines for colorectal cancer reporting, follows a similar pattern to the RCRG. It categorises tumours as having no residual tumour cells, minimal residual tumour or no marked regression. ${ }^{24}$ For ease of comparison, table 1 summarises the key features of the pathological classification systems proposed to date.

\section{TRG and clinical outcomes}

Until recently, there was a general debate about whether pathological measures of tumour regression correlated with overall survival from rectal cancer treated with neoadjuvant

Table 1 A summary of the commonly used systems for assessing rectal tumour regression

Dworak et $a l^{22}$

GR 0

GR 1

GR 2

GR 3

GR 4

No regression

Dominant tumour mass with obvious fibrosis and/or vasculopathy

Dominant fibrotic changes with few tumour cells or groups (easy to find)

Very few (difficult to find microscopically) tumour cells in fibrotic tissue with or without mucous substance

Rectal Cancer Regression Grading (RCRG) system

RCRG 1

Sterilisation or only microscopic foci of adenocarcinoma remaining, with marked fibrosis

RCRG 2 Marked fibrosis but macroscopic disease present

RCRG 3 Little or no fibrosis, with abundant macroscopic disease

RCPath dataset for colorectal cancer

RCPath A

RCPath B

No residual tumour cells and/or mucus lakes only Minimal residual tumour, that is, only occasional microscopic foci are identified with difficulty

RCPath C No marked regression 
chemoradiotherapy followed by surgery. ${ }^{25}$ Two recent papers have provided compelling evidence to support TRG as an independent predictor of survival. Maas et al published a metaanalysis of 3105 patients from 14 different study datasets who received neoadjuvant chemoradiotherapy followed by surgery. Of these, 484 patients had pathological complete regression (pCR). The group with pCR had more clinically and radiologically staged T1 or T2 tumours than those without pCR $(p<0.0001)$. At 5 years, those with $p C R$ had improved diseasefree survival ( $83.3 \%$ vs $65.6 \%, p<0.0001)$; lower risk of localised recurrence $(2.8 \%$ vs $9.7 \%, p<0.0001)$; better chance of being free from distant metastasis (88.8\% vs $74.9 \%, p<0.0001)$; and increased overall survival $(87.6 \%$ vs $76.4 \%, p<0.0001)$. The benefit of pCR is confirmed on multivariate analysis, with the HR for disease-free survival being $0.54(0.40-0.73)$ and that for overall survival being $0.65(0.7-0.89)$ in the group exhibiting pCR. ${ }^{26}$ These results are supported by the MERCURY study investigators, who reported that a ypT0 resection following neoadjuvant chemoradiotherapy was associated with increased disease-free and overall survival, as well as decreased rates of local recurrence. ${ }^{14}$

Following these two reports, the general consensus among clinicians now is that $\mathrm{pCR}$ is an independent predictor of the likelihood of local recurrence, distant metastasis, overall and disease-free survival and it is therefore a potential tool to guide therapy in patients with rectal cancer.

\section{TOWARDS A UNIVERSALLY ACCEPTED SYSTEM FOR TRG The need for consensus}

The development of TRG for the assessment of response to preoperative chemoradiotherapy in rectal cancer is hampered by the current lack of a universally accepted grading system. Bateman et al investigated the utility and reproducibility of the three commonly used scoring systems discussed above: TRG, RCRG and the Royal College of Pathologists systems. They modified the RCRG by avoiding the assessment of macroscopic features, instead defining the new grading entirely according to microscopic features. m-RCRG 1 had no tumour epithelium or scattered foci of malignant epithelium representing $<5 \%$ of the overall area of abnormality; in m-RCRG 2 malignant epithelium comprises $5 \%-50 \%$ of the overall area of abnormality; and m-RCRG 3 is defined as having over $50 \%$ of the area of abnormality comprising malignant epithelium. They found that all three systems were reproducible, with good inter-observer variability. $^{27}$

One of the key research questions yet to be answered is whether partial tumour regression is, like pCR, associated with better long-term outcomes. Despite a large number of studies examining this question, only two have shown lesser degrees of tumour regression to be prognostic factors on multivariate analysis. $^{28} 29$ In one of these studies (Min et al), partial tumour regression was only found to predict progression in lymph node negative rectal cancers. ${ }^{28}$ Due to the lack of standardisation of the way the specimen is analysed, the various reporting schemes utilised and the lack of inter-observer reproducibility for those patients with an incomplete response to therapy, a definitive conclusion to the debate about the significance of lesser grades of regression does not look likely at present. ${ }^{30}$ There is a clear need for international agreement both on a standardised method of specimen analysis and a reliable and reproducible way to score the presence of residual tumour. One such approach has been proposed recently following intensive discussion in the international pathology community. ${ }^{31}$

\section{Impact on surgical strategies}

Surgical strategies for the management of rectal cancers increasingly concentrate on the promotion of sphincter-sparing techniques. The use of localised excision with transanal endoscopic microsurgery for early (T1) rectal cancers is becoming increasingly accepted. It is, however, important to identify those patients who have unfavourable pathology that makes them at a high risk of local recurrence. It has been demonstrated that an aggressive strategy of early salvage surgery in transanal endoscopic microsurgery resections yielding high-risk pT1 tumours (those which were high grade, G3/4, those exhibiting lymphatic or venous involvement, or threatened/involved circumferential resection margins) and all pT2 tumours improves both tumourfree and tumour-related survival. ${ }^{32}$

The role of neoadjuvant therapy prior to localised excision is currently a highly active area of clinical research. It is thought that there is a risk of overtreating patients who could be cured by resection alone. ${ }^{33}$ The combination of neoadjuvant therapy and localised excision, however, provides a potential alternative in patients, with more advanced rectal tumours, who are unwilling or unable to undergo more extensive surgery. It has been shown that patients with radiological T3/T4 tumours exhibiting a pCR to neoadjuvant chemoradiotherapy are at a low risk of local recurrence. ${ }^{12}$ A small study (26 patients) looking at neoadjuvant chemoradiotherapy followed by local excision of the tumour for more advanced (T2/T3) distal rectal tumours with good clinical response to preoperative therapy used pCR to predict recurrence. In the short follow-up (mean follow-up 24 months), there were no recurrences in the group exhibiting pCR. Those patients who did not show pCR were offered salvage resection. The only patient with recurrence refused abdomino-perineal resection after partial response to neoadjuvant treatment. ${ }^{34}$ Thus, pCR is potentially a useful tool in this patient group as it allows the combination of neoadjuvant chemoradiotherapy and minimally invasive initial surgery, followed by early salvage surgery if required for more advanced tumours.

\section{The use of adjuvant chemotherapy}

In addition to its potential impact on surgical strategies, pathological regression grading can be used to guide adjuvant therapy and the intensity of post-treatment surveillance. There is much current interest in factors that can be used to guide choice of adjuvant therapy and surveillance following treatment of rectal cancer. This has resulted in the recent publication of nomograms to predict local recurrence, distant metastases and survival for patients with LARC treated with long-course chemoradiotherapy. ${ }^{35}$ Valentini and colleagues identified pathological tumour stage as a significant factor for prediction of 5-year probability of local recurrence, distant metastasis and overall survival. In their analysis, pT0 tumours were associated with better outcomes than those with worse pathological stages. ${ }^{35}$

\section{Long-term surveillance}

A 10-year follow-up study of 297 consecutive patients receiving neoadjuvant chemoradiotherapy in the USA showed that rectal cancer recurrence may be delayed following neoadjuvant therapy and resection, but that in the 44 patients (15\%) showing $\mathrm{pCR}$, only one patient suffered disease recurrence and no recurrence happened after the first 2 years of follow-up. This finding suggests that patients exhibiting pCR may need a shorter period of follow-up than those with lesser degrees of pathological response. ${ }^{36}$ The ability to correctly identify pCR 


\section{Take-home messages}

- Pathological complete response is an independent predictor of outcome following neoadjuvant chemoradiotherapy for locally advanced rectal cancer.

- There is currently a lack of consensus on how to classify degrees of tumour regression less than a complete pathological response in a system that could guide clinical management.

- The clinical validation of a universally accepted regression scoring system is a key research priority.

may therefore help to determine frequency and duration of follow-up and potential benefit from adjuvant chemotherapy.

Another area of current clinical research in the treatment of LARC is the avoidance of surgery in patients exhibiting a complete radiological response to neoadjuvant chemoradiation on post-treatment restaging. The potential feasibility of this approach has been demonstrated by Habr-Gama et al in South America ${ }^{37}$ and it is currently being tested in a prospective, multicentre National Cancer Research Network phase II clinical study in the $\mathrm{UK}^{38}$

\section{CONCLUSIONS}

Generally speaking, $\mathrm{pCR}$ is now accepted as an independent predictor of long-term outcomes following neoadjuvant chemoradiotherapy for LARC. Further work is needed, however, to determine the clinical importance of lesser degrees of pathological regression. A robust and internationally accepted system for the grading of tumour regression in rectal cancer following neoadjuvant chemoradiotherapy is currently required. Such consistency will help with clinical decision-making and will influence surgical strategies, postoperative adjuvant therapy and surveillance intensity. In the future, an accurate system of tumour regression, which has been shown to correlate with clinical outcome, may be used to avoid unnecessary treatment for some patients, while allowing patients at a greater risk of tumour recurrence to be offered more intensive therapy. The development of a universally accepted scoring system for TRG with well-validated correlates to clinical outcomes is a key research priority.

Acknowledgements RAS and TSM are funded by the NIHR Biomedical Research Centre Oxford, the Higher Education Funding Council for England, the UK Medical Research Council and Cancer Research UK.

Contributors All authors contributed to the design, writing and review of the article prior to submission.

\section{Competing interests None.}

Provenance and peer review Commissioned; internally peer reviewed.

\section{REFERENCES}

1. Quirke $\mathbf{P}$, Dixon MF. The prediction of local recurrence in rectal adenocarcinoma by histopathological examination. Int J Colorectal Dis 1988;3:127-31.

2. MacFarlane JK, Ryall RD, Heald RJ. Mesorectal excision for rectal cancer. Lancet 1993:341:457-60.

3. Beets-Tan RG, Beets GL, van der Hoop AG, et al. High-resolution magnetic resonance imaging of the anorectal region without an endocoil. Abdom Imaging 1999;24:576-81.

4. Brown G, Richards CJ, Newcombe RG, et al. Rectal carcinoma: thin-section MR imaging for staging in 28 patients. Radiology 1999;211:215-22.

5. Gerard A, Buyse M, Nordlinger B, et al. Preoperative radiotherapy as adjuvant treatment in rectal cancer. Final results of a randomized study of the European
Organization for Research and Treatment of Cancer (EORTC). Ann Surg 1988;208:606-14.

6. Frykholm GJ, Glimelius B, Pahlman L. Preoperative or postoperative irradiation in adenocarcinoma of the rectum: final treatment results of a randomized trial and an evaluation of late secondary effects. Dis Colon Rectum 1993;36:564-72.

7. Glimelius B, Isacsson U. Preoperative radiotherapy for rectal cancer-is $5 \times 5$ Gy a good or a bad schedule? Acta Oncol 2001;40:958-67.

8. van Gijn W, Marijnen CA, Nagtegaal ID, et al. Preoperative radiotherapy combined with total mesorectal excision for resectable rectal cancer: 12-year follow-up of the multicentre, randomised controlled TME trial. Lancet Oncol 2011;12:575-82.

9. Pettersson D, Cedermark B, Holm T, et al. Interim analysis of the Stockholm III trial of preoperative radiotherapy regimens for rectal cancer. Br J Surg 2010;97:580-7.

10. Prolongation of the disease-free interval in surgically treated rectal carcinoma. Gastrointestinal Tumor Study Group. N Engl J Med 1985;312:1465-72.

11. Krook JE, Moertel CG, Gunderson LL, et al. Effective surgical adjuvant therapy for high-risk rectal carcinoma. N Engl J Med 1991;324:709-15.

12. Sauer R, Becker $\mathrm{H}$, Hohenberger $\mathrm{W}$, et al. Preoperative versus postoperative chemoradiotherapy for rectal cancer. N Engl J Med 2004;351:1731-40.

13. Edge SB. American Joint Committee on Cancer. AJCC Cancer Staging Manual. 7th edn. New York, London: Springer, 2010.

14. Patel UB, Taylor F, Blomqvist L, et al. Magnetic resonance imaging-detected tumo response for locally advanced rectal cancer predicts survival outcomes: MERCURY experience. J Clin Oncol 2011;29:3753-60.

15. Shumate CR, Rich TA, Skibber JM, et al. Preoperative chemotherapy and radiation therapy for locally advanced primary and recurrent rectal carcinoma. A report of surgical morbidity. Cancer 1993;71:3690-6.

16. Cassidy J, Saltz L, Twelves C, et al. Efficacy of capecitabine versus 5 -fluorouracil in colorectal and gastric cancers: a meta-analysis of individual data from 6171 patients. Ann Oncol 2011;22:2604-9.

17. Ramani VS, Sun Myint A, Montazeri A, et al. Preoperative chemoradiotherapy for rectal cancer: a comparison between intravenous 5 -fluorouracil and oral capecitabine. Colorectal Dis 2010;12(Suppl 2):37-46.

18. Gollins S, Sun Myint A, Haylock B, et al. Preoperative chemoradiotherapy using concurrent capecitabine and irinotecan in magnetic resonance imaging-defined locally advanced rectal cancer: impact on long-term clinical outcomes. J Clin Oncol 2011;29:1042-9

19. Hill EJ, Nicolay NH, Middleton MR, et al. Oxaliplatin as a radiosensitiser for upper and lower gastrointestinal tract malignancies: what have we learned from a decade of translational research? Crit Rev Oncol Hematol. Published Online First: 4 February 2012. doi:10.1016/j.critrevonc.2011.12.007

20. Velenik V, Ocvirk J, Oblak I, et al. A phase II study of cetuximab, capecitabine and radiotherapy in neoadjuvant treatment of patients with locally advanced resectable rectal cancer. Eur J Surg Oncol 2010;36:244-50.

21. Mandard AM, Dalibard F, Mandard JC, et al. Pathologic assessment of tumor regression after preoperative chemoradiotherapy of esophageal carcinoma. Clinicopathologic correlations. Cancer 1994;73:2680-6.

22. Dworak 0, Keilholz L, Hoffmann A. Pathological features of rectal cancer after preoperative radiochemotherapy. Int J Colorectal Dis 1997;12:19-23.

23. Wheeler JM, Warren BF, Mortensen NJ, et al. Quantification of histologic regression of rectal cancer after irradiation: a proposal for a modified staging system. Dis Colon Rectum 2002;45:1051-6.

24. Williams JT, Quirke P, Shepherd NA. Dataset for Colorectal Cancer. 2nd edn. London: The Royal College of Pathologists, 2007. (Updated September 2007). http:// www.rcpath.org/Resources/RCPath/Migrated\%20Resources/Documents/G/G049ColorectalDataset-Sep07.pdf (accessed May 2012).

25. Pucciarelli S, Toppan P, Friso ML, et al. Complete pathologic response following preoperative chemoradiation therapy for middle to lower rectal cancer is not a prognostic factor for a better outcome. Dis Colon Rectum 2004;47:1798-807.

26. Maas M, Nelemans PJ, Valentini V, et al. Long-term outcome in patients with a pathological complete response after chemoradiation for rectal cancer: a pooled analysis of individual patient data. Lancet Oncol 2010:11:835-44.

27. Bateman AC, Jaynes E, Bateman AR. Rectal cancer staging post neoadjuvant therapy-how should the changes be assessed? Histopathology 2009;54:713-21.

28. Min BS, Kim NK, Pyo JY, et al. Clinical impact of tumor regression grade after preoperative chemoradiation for locally advanced rectal cancer: subset analyses in lymph node negative patients. J Korean Soc Coloproctol 2011;27:31-40.

29. Lin CY, Tian YF, Wu LC, et al. Rsf-1 expression in rectal cancer: with special emphasis on the independent prognostic value after neoadjuvant chemoradiation. J Clin Pathol. Published Online First: 8 May 2012. doi:10.1136/jclinpath-2012-200786

30. Chetty R, Gill P, Govender D, et al. A multi-centre pathologist survey on pathological processing and regression grading of colorectal cancer resection specimens treated by neoadjuvant chemoradiation. Virchows Arch 2012;460:151-5.

31. Chetty R, Gill P, Govender D, et al. International study group on rectal cancer regression grading: interobserver variability with commonly used regression grading systems. Hum Pathol. Published Online First: 8 May 2012. doi:10.1016/ j.humpath.2012.01.020

32. Borschitz T, Gockel I, Kiesslich R, et al. Oncological outcome after local excision of rectal carcinomas. Ann Surg Oncol 2008;15:3101-8.

33. Tytherleigh MG, Warren BF, Mortensen NJ. Management of early rectal cancer. $B$ J Surg 2008:95:409-23.

34. Kim CJ, Yeatman TJ, Coppola D, et al. Local excision of T2 and T3 rectal cancers after downstaging chemoradiation. Ann Surg 2001;234:352-8. 
35. Valentini V, van Stiphout RG, Lammering G, et al. Nomograms for predicting local recurrence, distant metastases, and overall survival for patients with locally advanced rectal cancer on the basis of European randomized clinical trials. $J$ Clin Oncol 2011:29:3163-72.

36. Guillem JG, Chessin DB, Cohen AM, et al. Long-term oncologic outcome following preoperative combined modality therapy and total mesorectal excision of locally advanced rectal cancer. Ann Surg 2005;241:829-36.
37. Habr-Gama A, Perez RO, Nadalin W, et al. Operative versus nonoperative treatment for stage 0 distal rectal cancer following chemoradiation therapy: long-term results. Ann Surg 2004;240:711-17.

38. Chong IY, Brown G, Heald RJ, et al. A multicenter phase II clinical study evaluating the deferral of rectal surgery following a continued response to pre-operative chemoradiotherapy (CRT) J Clin Oncol 2010:28: TPS191. 